This item was submitted to Loughborough's Research Repository by the author.

Items in Figshare are protected by copyright, with all rights reserved, unless otherwise indicated.

\title{
All talk? Challenging the use of left-temporal EEG alpha oscillations as valid measures of verbal processing and conscious motor control
}

\section{PLEASE CITE THE PUBLISHED VERSION}

https://doi.org/10.1016/j.biopsycho.2020.107943

\section{PUBLISHER}

Elsevier BV

VERSION

AM (Accepted Manuscript)

\section{PUBLISHER STATEMENT}

This paper was accepted for publication in the journal Biological Psychology and the definitive published version is available at https://doi.org/10.1016/j.biopsycho.2020.107943

LICENCE

CC BY-NC-ND 4.0

\section{REPOSITORY RECORD}

Parr, Johnny VV, Germano Gallicchio, Neil R Harrison, Ann-Kathrin Johnen, and Greg Wood. 2020. "All Talk? Challenging the Use of Left-temporal EEG Alpha Oscillations as Valid Measures of Verbal Processing and Conscious Motor Control”. Loughborough University. https://hdl.handle.net/2134/12792383.v1. 


\section{Journal Pre-proof}

All talk? Challenging the use of left-temporal EEG alpha oscillations as valid measures of verbal processing and conscious motor control

Johnny V.V. Parr, Germano Gallicchio, Neil R. Harrison, Ann-Kathrin Johnen, Greg Wood

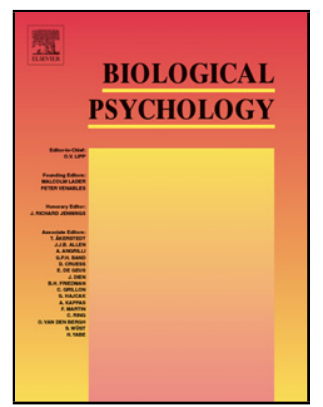

PII: S0301-0511(20)30103-4

DOI: $\quad$ https://doi.org/10.1016/j.biopsycho.2020.107943

Reference: BIOPSY 107943

To appear in: $\quad$ Biological Psychology

Received Date: $\quad 24$ October 2019

Revised Date: $\quad 19$ May 2020

Accepted Date: $\quad 27$ July 2020

Please cite this article as: Parr JVV, Gallicchio G, Harrison NR, Johnen A-Kathrin, Wood G, All talk? Challenging the use of left-temporal EEG alpha oscillations as valid measures of verbal processing and conscious motor control, Biological Psychology (2020), doi: https://doi.org/10.1016/j.biopsycho.2020.107943

This is a PDF file of an article that has undergone enhancements after acceptance, such as the addition of a cover page and metadata, and formatting for readability, but it is not yet the definitive version of record. This version will undergo additional copyediting, typesetting and review before it is published in its final form, but we are providing this version to give early visibility of the article. Please note that, during the production process, errors may be discovered which could affect the content, and all legal disclaimers that apply to the journal pertain.

(c) 2020 Published by Elsevier. 
All talk? Challenging the use of left-temporal EEG alpha oscillations as valid measures of verbal processing and conscious motor control

Johnny V. V. Parr ${ }^{1}$, Germano Gallicchio ${ }^{2}$, Neil R. Harrison ${ }^{3}$, Ann-Kathrin Johnen ${ }^{4}$, \& Greg Wood ${ }^{1}$.

1. Research Centre for Musculoskeletal Science and Sports Medicine, Department of Sport and Exercise Sciences, Manchester Metropolitan University, Manchester, UK.

2. School of Sport, Exercise and Health Sciences, Loughborough University, Loughborough, UK.

3. Department of Psychology, Liverpool Hope University, Liverpool, UK.

4. School of Psychology, University of East Anglia, Norfolk, UK

\section{Address correspondence to:}

Johnny V. V. Parr, PhD.

Research Centre for Musculoskeletal Science and Sports Medicine,

Department of Sport and Exercise Sciences,

Manchester Metropolitan University,

Manchester, M1 5DG, UK

Email: j.parr@mmu.ac.uk 


\title{
Highlights
}

- Task-unrelated self-talk had no effect on left temporal alpha power

- Task-related self-talk decreased alpha power across entire scalp

- T7-Fz connectivity was unresponsive to self-talk manipulations

\begin{abstract}
This study tested the validity of EEG left-temporal alpha power and upper-alpha T7-Fz connectivity as indices of verbal activity and conscious motor control. Participants $(n=20)$ reached for, and transported, a jar under three conditions: a control condition and two self-talk conditions aimed at eliciting either task-unrelated verbal processing or task-related conscious control, while EEG and hand kinematics were recorded. Compared to the control condition, both self-talk conditions increased self-reported verbal processing, but only the task-related self-talk condition increased lefttemporal activity (i.e., alpha power decreased). However, as cortical activity increased across the entire scalp topography, conscious control likely elicits a multitude of processes that may not be explained by left-temporal activity or verbal processing alone, but by a widespread decrease in neural efficiency. No significant effects for T7-Fz connectivity were detected. Results suggest that lefttemporal EEG alpha oscillations are unlikely to uniquely reflect verbal processing during conscious motor control.
\end{abstract}

Keywords: conscious motor processing, reinvestment, verbal-analytical, $T 7-F z$, neural efficiency. 


\section{Introduction}

Motor skill acquisition is underpinned by fundamental changes in the knowledge structures used to support motor learning and effective performance. In the early stages of learning, performers are reliant on task-specific, declarative knowledge that is verbal and conscious in nature. This is often referred to as the verbal-cognitive stage of learning and is characterised by movements that are inefficient, effortful, slow, error-strewn, and inconsistent. As expertise develops, learners transition to a stage where declarative knowledge has become proceduralised and performance is free from verbal interference and is unconscious in nature. This is often referred to as the autonomous stage of learning and is characterised by movements that are efficient, effortless, errorless and consistent (Fitts \& Posner, 1967).

By using neuroimaging techniques, such as electroencephalography (EEG), researchers have examined changes in cerebral cortex activity in order to measure the contribution of declarative knowledge and conscious movement control during motor skill acquisition. A cortical region that is thought to be implicated in this process is the left-temporal region. By recording the magnitude of EEG oscillatory activity within the $8-12 \mathrm{~Hz}$ frequency range - an inverse marker of neuronal activity termed "alpha power" (Klimesch, 2012; Jensen \& Mazaheri, 2010) - researchers have observed diminished activity in the left-temporal region as a function of expertise (Haufler, Spalding, Santa Maria, \& Hatfield, 2000; Janelle et al., 2000), training (Kerick, Douglass, \& Hatfield, 2004), and performance (Gallicchio, Cooke, \& Ring, 2017). As the left-temporal region includes structures implicated in language processing (e.g., Broca's area and Wernicke's area), these findings have been interpreted as evidence that expert performance is less reliant on declarative verbal processes that characterise the conscious motor control of novices.

In addition to regional activation, cortico-cortical networking has been examined to reveal the interaction across various cortical regions. For example, phase-based measures of alpha connectivity between the left-temporal (T7) and the frontal premotor region $(\mathrm{Fz})$ have been examined to assess the influence of declarative, verbal processing on the production of movement. Alpha connectivity reflects the synchronicity in the inhibition profiles of two regions, with greater alpha connectivity 
suggesting consistently similar inhibition (functional communication) and lower alpha connectivity suggesting distinct inhibition profiles (regional independence). In line with the acquisition of greater automaticity with training, researchers have shown that T7-Fz upper-alpha $(\sim 10-12 \mathrm{~Hz})$ connectivity decreases with increasing skill (Deeny, Hillman, Janelle, \& Hatfield, 2003; Gallicchio, Cooke, \& Ring, 2016; Gallicchio et al., 2017; Kerick et al., 2004). This is suggestive of a gradual disconnect between the verbal and premotor regions as individuals consolidate movement patterns and efficiently organise task-related neural networks free from conscious control. Further work has shown that T7-Fz upper-alpha connectivity increases when directing participants to exert conscious movement control (Ellmers et al., 2016) and is greater for novices who undertake explicit motor learning (high exposure to declarative knowledge) compared to those who undertake implicit motor learning (low exposure to declarative knowledge) (Parr, Vine, Wilson, Harrison, \& Wood, 2019; Zhu, Poolton, Wilson, Hu, et al., 2011). Finally, participants who report a strong propensity to consciously monitor and control their movements also display increased T7-Fz connectivity compared with those with a lower propensity (Zhu, Poolton, Wilson, Maxwell, \& Masters, 2011).

Taken together, these findings suggest that skilled, autonomous, expert-like motor performance is associated with decreased left-temporal involvement in the production of movement. Conversely, less skilled, conscious, and novice-like motor performance is associated with increased left-temporal involvement in the production of movement. Left-temporal alpha oscillations would therefore appear a useful index for the verbal-cognitive processes that underpin conscious motor control.

Implicit within this proposition is the assumption that EEG activity recorded over lefttemporal sites (i.e., T7) during motor execution is representative of the underlying cortical structures associated with language (Cooke, 2013). However, this may not be the case for two reasons: first, unless a one-to-one mapping can be demonstrated, it is not deductively valid to infer a particular cognitive process from the activation of a particular brain region. This practice, termed "reverse inference", reasons backwards from the presence of brain activation to the engagement of a particular cognitive function, and is limited by non-selectivity of activation in the region of interest (Poldrack, 
2006). For example, structures across the left-temporal region are implicated not only in language processing but also in auditory processing (Tiihonen et al., 1991) and the integration of visual and auditory information (Beauchamp, Lee, Argall, \& Martin, 2004). Second, the EEG has a relatively low spatial resolution due to the propagation of electrical fields across tissues - a phenomenon referred to as "volume conduction" - meaning that the activity recorded from channels located over the left-temporal region can detect not just local (underlying) sources but also large distal sources (Cohen, 2015). For example, right-handed motor actions will elicit the activation of the left precentral gyrus (Pfurtscheller \& Aranibar, 1979; Pfurtscheller \& Berghold, 1989) that has been shown to elicit alpha power desynchronization across central, frontal and temporal regions (Harmon-Jones, 2006).

It is therefore unclear the extent to which EEG alpha activity recorded over left-temporal sites may reflect a broader range of processes associated with conscious motor control beyond that of language, such as increased demand on attentional resources and increased cognitive and motor effort. This issue is corroborated by research showing that, compared to experts, novices display a global decrease in alpha power - termed neural efficiency - that is not specific to the left-temporal region (Babiloni et al., 2010; Baumeister, Reinecke, Liesen, \& Weiss, 2008; Del Percio et al., 2009; Janelle et al., 2000; Parr et al., 2019). It is therefore plausible that differences in left-temporal alpha during conscious motor control are more reflective of general reductions in neural efficiency and increased effort, rather than reflective of the involvement in verbal processing that is often assumed to occur.

The aim of this study was to manipulate self-talk to explore how verbal processing and conscious control affect measures of regional alpha power and connectivity during movement production. Specifically, we compared task-related and task-unrelated self-talk with uninstructed, natural performance during reaching and grasping movements. Whilst both types of self-talk were administered to increase verbal processing, only task-related self-talk was designed to induce conscious motor control that would increase cognitive effort (Masters, 1992). We hypothesised that if left-temporal alpha oscillations reflect verbal processing, then both conditions that encourage self-talk (task-related and task-unrelated) would increase left-temporal activity (decreased alpha power) and left temporal frontal (T7-Fz) connectivity during the motor task. Conversely, if left-temporal alpha 
oscillations were specifically related to conscious movement control, then we would only expect increased left-temporal activity, and increased left temporal-frontal connectivity, during the effortful, task-related self-talk condition. More broadly, if conscious movement control induces changes in regions other than left-temporal, this would support the thesis that the association between lefttemporal activity and conscious movement control can be attributed to a spatially broader phenomenon associated with cognitive processes beyond language alone, possibly consistent with decreased neural efficiency.

\section{Methods}

\subsection{Participants}

Twenty self-reported right-handed participants took part in the study (11 females, 9 males, age: $M=26.38, S D=6.19$ years). A-priori sample size calculation was performed using $\mathrm{G}^{*}$ Power 3.1.9.2 (Faul, Erdfelder, Buchner, \& Lang, 2009) based on previous work in upper limb control (Parr et al., 2019), comparing EEG alpha power and T7-Fz connectivity after explicit or implicit prosthetic hand training. To detect an effect size of $\eta p 2=0.145$, with an alpha of .05 , a sample size of at least 12 were required for our repeated measures design to yield $80 \%$ power. We chose to recruit 20 participants to account for potential data loss prevalent in EEG data. All participants gave written consent and the procedures were approved by our institutional ethics committee.

\subsection{Experimental task}

The task required participants to sequentially reach for and then transport a glass jar from a home position to a target position on a desk (Figure 1). Prior to each trial, participants placed their right hand on the start location positioned at the desk edge approximately $30 \mathrm{~cm}$ away from the jar home position. The trial started with the onset of an auditory tone (S1) indicating the start of a 2second preparation period. During this period, participants were instructed to stay still, maintain their hand on the start location, focus their gaze on the jar, and avoid eye blinks and body movement to minimise EEG data artefacts. At the end of this 2-second period, a second auditory tone (S2) acted as a "go" signal to initiate the task. After placing the jar on the target position, participants returned their 
hand to the start location and the researcher prepared the workspace for the following trial.

Participants were instructed to perform at a steady speed that felt comfortable and natural. As previous research has shown reaching and grasping with additional weights of $1 \mathrm{~kg}$ has no effect on EEG alpha activity (Parr, 2018), the weight of the jar was randomly varied at each trial among five options $(250,350,450,550,650 \mathrm{~g})$ to decrease the repetitive nature of the task and increase task engagement. Auditory tones (each 0.67 seconds long) were controlled through E-Prime 2.0 (Psychology Software Tools, Inc., Pittsburgh, PA, USA).

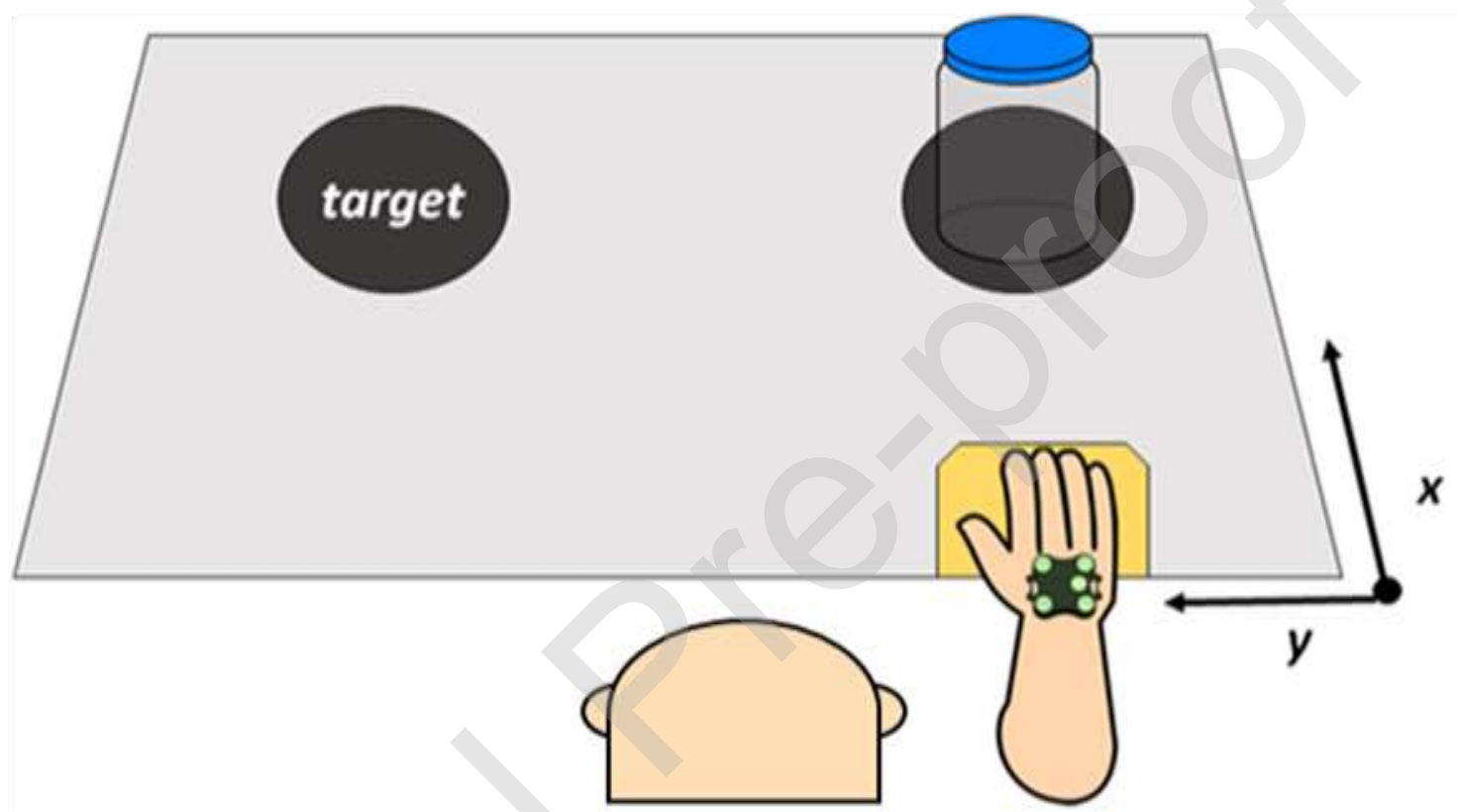

Figure 1. Visual representation of the experimental set-up. Participants began each trial with their right hand on the start position (in yellow). A first auditory tone (S1) indicated the start of the 2second preparation period, during which participants maintained their hand on the start position and their gaze on the jar. Following a second auditory tone (S2) participants reached for the jar and placed it on the target location.

\subsection{Procedure}

Participants attended one 2-hour session that involved participant preparation, electrode placement, and the completion of each experimental condition. After briefing, participants sat at a distance that enabled them to reach the jar home and target positions at arms-length. Once 
instrumented for EEG and kinematic recording, participants completed a 1-minute eyes-closed resting baseline and then completed 10 practice trials to enable familiarise the task. Participants then performed 40 trials in the control condition, which consisted of reaching and transporting the jar with no self-talk instructions. Participants then completed manipulation checks at the end of the 40 trials assessing task difficulty, mental load, conscious control, and self-talk (see below for details).

The experiment was then performed under two self-talk conditions - one task-related and one task-unrelated - each composed of 40 trials. These trials were performed in eight blocks of ten in an interleaved manner (e.g., $10 \times$ task-related, $10 \times$ task-unrelated, $10 \times$ task-related etc.), with the starting condition counterbalanced across participants. For the task-related condition, self-talk instructions were designed to encourage conscious motor control: "Keep elbow below the wrist", "Keep palm 5(cm) from table", "Keep thumb below the index", "Keep wrist flexed at 90 degrees". For the task-unrelated condition, self-talk instructions included a collection of well-known nursery rhymes that were unrelated to movement: "Jack and Jill went up the hill", "Twinkle twinkle little star", "Mary had a little lamb", "Humpty dumpty sat on a wall". Only one (randomly selected) self-talk instruction was administered for each new block of ten trials. Participants were required to silently rehearse this instruction throughout the entirety of each trial (i.e., during both the planning $(P)$ and movement $(M)$ task phases) to the best of their ability. For both self-talk conditions, participants were asked which word of the given phrase they finished on when placing the jar. This word was written down in view of each participant to act as a manipulation check and to encourage adherence to selftalk instructions. Adherence to the task-related self-talk condition was further encouraged by positioning electrodes on the right upper-limb and workspace prior to each block of 10 trials to convince participants that their adherence to the task-related instructions would be quantitatively evaluated (Appendix 1). After each of the ten blocks of trials, participants again rated task difficulty, mental load, conscious control, and self-talk (see below for details).

\subsection{EEG}

Thirty-two active EEG electrodes were positioned on the scalp at locations Fp1, Fp2, AF3, AF4, F7, F3, Fz, F4, F8, FC5, FC1, FC2, FC6, T7 (T3), C3, Cz, C4, T8 (T4), CP5, CP1, CP2, CP6, 
P7, P3, Pz, P4, P8, PO3, PO4, O1, Oz and O2 of the 10-20 system (Jasper, 1958) ${ }^{1}$. Common mode sense (CMS) and driven right leg (DRL) electrodes were used to enhance the common mode rejection ratio of the signal. The signal was amplified and digitized at $512 \mathrm{~Hz}$ using the ActiveTwo recording system (Biosemi, The Netherlands). A digital trigger was automatically sent from E-Prime to the Biosemi system via parallel communication at the onset of each S2 ("go" signal). All EEG signals were re-referenced to the average of all channels and band-pass filtered from $1 \mathrm{~Hz}$ (low-pass) to 35 $\mathrm{Hz}$ (high-pass) using EEGLAB's basic FIR (finite infinite response) filter. Epochs were cut from 2.25 to +2.25 s relative to the onset of S2. Epochs were then visually inspected so those showing movement artefact could be rejected (percentage of trials rejected: Control, $M=5 \%, S D=6 \%$, Taskrelated, $M=6 \%, S D=7 \%$, Task-unrelated, $M=8 \%, S D=6 \%$ ). No bad channels were identified. Data were then subjected to Independent Component Analysis (Runica Infomax algorithm; Makeig, Bell, Jung, \& Sejnowski, 1996) to identify and remove components accounting for blinks, eye movements and other non-neural activity (components removed: $M=5.70, S D=3.05$ ). These processing steps were performed using EEGLAB functions (v9.4.0.813654, Delorme \& Makeig, 2004) and bespoke MATLAB scripts.

\subsection{Kinematic data}

Noraxon MyoMotion (Scottsdale, AZ, USA) motion analysis system was employed to analyse kinematic characteristics of the task performance. A single MyoMotion inertial measurement unit (IMU) was placed on the dorsal side of the right hand according to the rigid-body model defined in the Noraxon MR3 software. Calibration was performed using the upright standing position, though the use of a single IMU wavered the need for a zero/neutral angle in the measured joints. The sampling frequency for the inertial sensor was set at $100 \mathrm{~Hz}$. To identify movement onset (S2) for each trial, a tripod-mounted webcam $(30 \mathrm{~Hz})$ was synchronised using a light-emitting diode (LED) and Noraxon software to enable digital triggers to be applied to the data offline following visual

\footnotetext{
${ }^{1}$ Sites T7 and T8 are sometimes referred to as T3 and T4, respectively, in other EEG systems.
} 
inspection of the recorded video. Data were then smoothed using a 10-point (corresponding to 100ms) moving average before being epoched from -2.25 to +2.25 s relative to S2 ("go" signal) for each trial.

\subsection{Measures}

\subsubsection{Manipulation check}

Manipulation checks were administered throughout testing to confirm that our experimental conditions were having their desired effect. Participants were asked to self-report levels of task difficulty ("How difficult was it to move the jar accurately?"); mental effort ("How much mental effort did you exert whilst performing?"); movement awareness ("How often were you aware of your hand and arm movements?"); movement control ("How often were you trying to control your hand and arm movements?"); self-talk frequency (“How often did you experience self-talk?") and self-talk intensity (“How intense and clear was your self-talk?"). Responses were given on a 7-point Likert scale ranging from 1 (no effort/never/not intense) to 7 (extreme effort/always/extremely intense). Selfreport responses were given via a computer. Data from one participant was corrupt and therefore removed from analyses.

\subsubsection{Movement time}

Movement time was measured as the time (in seconds) elapsed between S2 (i.e., "go" signal) and jar placement on the target location. To obtain this, the researcher provided a keyboard response upon jar placement, enabling movement time to be extracted from E-Prime.

\subsubsection{Peak acceleration}

The early characteristics of reaching and grasping movements can provide useful insights into the extent to which movements are performed under predictive or online control. For example, increased limb acceleration indicates more ballistic and pre-planned movement, whereas decreased limb acceleration would suggest a more conservative approach with greater reliance on feedback to supervise ongoing action (Desmurget \& Grafton, 2000). Peak acceleration along the $x$ - and $y$-axes of movement was therefore measured for the reach and transport phases of our task to provide kinematic evidence of how our self-talk instructions interfered with movement automaticity. Specifically, peak 
reach acceleration was taken as the peak value recorded between 0 and +500 ms relative to $S 2$ along the $x$-axis of movement. Peak transport acceleration was taken as the peak value recorded between 500 and 1500 ms relative to S2 along the $y$-axis of movement. Separate axes were chosen for each phase as they each capture the primary direction of movement. Time windows were selected upon visual inspection of data from which clear peaks for each phase could be easily detected (see Figure 4).

\subsubsection{Alpha power}

Time-frequency decomposition of the epoched EEG signals was performed for each trial through short-time Fast Fourier Transform (FFT) conducted on 37 overlapping windows, each of $0.5 \mathrm{~s}$ ( $87.5 \%$ overlap), with central points ranging from -2 to +2 s. Prior to FFT, data points in each window were Hanning-tapered and zero-padded to reach $4 \mathrm{~s}$. This procedure generated complex-valued coefficients in the time-frequency plane with a precision of $111 \mathrm{~ms}$ and $0.25 \mathrm{~Hz}$, separately for each channel and trial. Alpha power was then computed as the squared amplitude within each participant's individual alpha frequency (IAF) band, identified using both the 'peak frequency' and 'centre of gravity' (CoG) methods (Corcoran, Alday, Schlesewsky, \& Bornkessel-Schlesewsky, 2018). To do this, we first attempted to identify a visible peak (between approximately $7-15 \mathrm{~Hz}$ ) from the mean EEG spectrum over occipito-parietal channels, recorded from the 1-minute eyes-closed baseline. When a single peak was not present (40\% of participants), IAF was instead taken as the power spectra weighted mean (CoG) between 7 and $15 \mathrm{~Hz}$ (Appendix 2). Subsequently, the alpha frequency band was denoted as IAF-2 to IAF+2. For one participant, baseline data was corrupted, resulting in alpha being denoted as the typical $8-12 \mathrm{~Hz}$ range. As no neutral baseline could be identified, non-normal distributions and inter-individual differences were dealt with by employing a median-scaled log transformation. This transformation was implemented by scaling all alpha power values for each participant (across all channels, trials, time-windows, and conditions) by the median alpha power value within that participant, before then employing a $10 \cdot \log 10$ transformation (Gallicchio,

Finkenzeller, Sattlecker, Lindinger, \& Hoedlmoser, 2016). Power was then averaged across overlapping time windows and across trials to provide mean values for each channel, separately from 
$-1.5 \mathrm{~s}$ to $-0.75 \mathrm{~s}\left(P^{-2}\right),-0.75 \mathrm{~s}$ to $0 \mathrm{~s}\left(P^{-1}\right), 0 \mathrm{~s}$ to $0.75 \mathrm{~s}\left(M^{+1}\right)$, and $0.75 \mathrm{~s}$ to $1.5 \mathrm{~s}\left(M^{-2}\right)$. To examine regional effects, seven regions of interest (ROI) were identified based on inspection of topographical maps: frontal (F3, Fz, F4), left-temporal (F7, FC5, T7), left central (FC1, C3, CP1), right central (FC2, C3, $\mathrm{CP} 2)$, right temporal (F8, FC6, T8), parietal (P3, Pz, P4) and occipital (O1, Oz, O2). Values within each ROI were averaged. Signal processing was performed in MATLAB.

\subsubsection{Alpha connectivity}

Functional connectivity was computed as the inter-site phase clustering (ISPC) over time (Cohen, 2014; Lachaux, Rodriguez, Martinerie, \& Varela, 1999). ISPC measures the phase lag consistency across time between two channels independently from their power and reflects functional connectivity between the oscillatory activity of two underlying cortical regions, with values ranging from 0 (no connectivity) to 1 (perfect connectivity). ISPC was calculated for each trial using bespoke Matlab scripts as, $\operatorname{ISPC}(f)=\left|n^{-1} \sum_{w=1}^{n} e^{i(\theta x(w, f)-\theta y(w, f))}\right|$, where $i$ is the imaginary operator; $\theta x$ and $\theta y$ are the phase angles of the recorded signal at two different scalp locations at FFT time

window $w$ and frequency $f ; e^{i(\theta x(w, f)-\theta y(w, f))}$ denotes a complex vector with magnitude 1 and angle $\theta x-\theta y ; n^{-1} \sum_{w=1}^{n}(\cdot)$ denotes averaging across the overlapping FFT time windows within each predefined epoch $\left(P^{-2}, P^{-1}, M^{+1}, M^{+2}\right)$; and $|\cdot|$ is the module of the average vector. Following previous research and our a priori hypotheses, we focused on left-temporal (T7) - frontal (Fz) ISPC in the upper-alpha (IAF to IAF+2) frequency band for our statistical analyses. Right temporal (T8) - frontal (Fz) upper-alpha connectivity was also analysed to verify the extent to which connectivity effects were localised to a given hemisphere. No baselines were used. Instead, to normalise their density distributions, ISPC were Fisher $Z$ transformed (inverse hyperbolic tangent); values could then range from 0 to $\infty$. Values were then averaged over trials and frequencies within the upper-alpha range (IAF to IAF+2 Hz) to yield estimates of alpha connectivity separately for each condition.

\subsection{Statistical analyses}

For self-report data, a one-way repeated measures ANOVA was performed to assess the effect of Condition (control, task-related, task-unrelated) on each of the six items (difficulty, mental 
effort, movement awareness, movement control, self-talk frequency, self-talk intensity). One-way repeated measures ANOVAs were also performed to assess the effect of Condition on movement time, peak reach acceleration and peak transport acceleration.

A 3 Condition $\times 4$ Epoch $\left(P^{-2}, P^{-1}, M^{+1}, M^{+2}\right)$ repeated measures ANOVA on the averaged alpha power over channels T7, F7 and FC5 was performed to specifically test the prediction that lefttemporal alpha power can be used to infer verbal processing. Following this, a 3 Condition $\times 4$ Epoch $\times 7$ ROI (left-temporal, frontal, left central, right central, parietal, right temporal, occipital) repeated measures ANOVA was conducted to evaluate the presence of wider regional effects of alpha power. Finally, a 3 Condition $\times 4$ Epoch $\times 2$ Hemisphere $(\mathrm{T} 7-\mathrm{Fz}, \mathrm{T} 8-\mathrm{Fz})$ repeated measures ANOVA was conducted to assess time-varying changes in left and right temporal-frontal alpha connectivity across Conditions. By comparing the $1.5 \mathrm{~s}$ preceding movement with the $1.5 \mathrm{~s}$ following movement, we can determine whether effects are specific to the preparation or execution of motor performance.

For all ANOVAs, Greenhouse-Geisser corrections were applied when sphericity was violated, and effect sizes were calculated using partial eta squared ( $\eta$ 2). All post hoc pairwise comparisons were adjusted using Bonferroni corrections to counteract the problem of multiple comparisons.

\section{Results}

\subsection{Manipulation checks}

Results from each one-way repeated measures ANOVA showed a significant main effect of Condition for self-reported task difficulty, $F(2,36)=66.04, p<.001, \eta \mathrm{p} 2=.786$, mental effort, $F(2$, $36)=49.37, p<.001, \eta \mathrm{p} 2=.733$, movement awareness, $F(2,36)=57.56, p<.001, \eta \mathrm{p} 2=.762$, conscious control, $F(2,36)=77.08, p<.001, \eta p 2=.811$, self-talk frequency, $F(2,36)=27.37, p<$ $.001, \eta p 2=.603$, and self-talk intensity, $F(2,36)=14.739, p<.001, \eta p 2=.450$. Post-hoc pairwise comparisons showed significantly higher levels of self-talk frequency $(p<.001)$ and self-talk intensity $(p<.001)$ during the task-related and task-unrelated self-talk conditions compared to the control condition, confirming the intended manipulation of verbal processing. Task difficulty, mental effort, movement awareness, and conscious control were rated significantly higher in the task-related 
condition compared to the task-unrelated and control conditions $(p<.001)$, but the task-unrelated condition as significantly more difficult than the control condition $(p=.013)$.

Table 1. Means and standard deviations of self-report and kinematic data, together with the results from each one-way repeated-measures ANOVA.

\begin{tabular}{lccccc}
\hline & $\begin{array}{c}\text { Control } \\
\text { M (SD) }\end{array}$ & $\begin{array}{c}\text { Task-related } \\
\text { M (SD) }\end{array}$ & $\begin{array}{c}\text { Task-unrelated } \\
\text { M (SD) }\end{array}$ & $\boldsymbol{F}$ & $\boldsymbol{\eta}$ p2 \\
\hline Difficulty & $1.05(0.23)$ & $3.33(1.09)$ & $1.50(0.62)$ & $66.04^{*}$ & .79 \\
Mental effort & $1.47(0.96)$ & $4.14(1.00)$ & $2.07(1.00)$ & $49.37 *$ & .73 \\
Movement awareness & $3.16(1.30)$ & $5.74(1.09)$ & $2.88(0.81)$ & $57.56^{*}$ & .76 \\
Movement control & $2.47(1.07)$ & $5.71(0.85)$ & $2.39(1.08)$ & $77.08^{*}$ & .81 \\
Self-talk frequency & $4.16(1.53)$ & $6.54(0.56)$ & $6.43(0.69)$ & $27.37 *$ & .60 \\
Self-talk intensity & $3.89(1.79)$ & $5.92(0.88)$ & $5.81(0.86)$ & $14.74^{*}$ & .45 \\
Reach $\left(\right.$ peak $\left.\mathrm{m} / \mathrm{s}^{2}\right)$ & $2.20(0.52)$ & $1.49(0.40)$ & $2.04(0.47)$ & $34.38^{*}$ & .86 \\
Transport $\left(\right.$ peak $\left.\mathrm{m} / \mathrm{s}^{2}\right)$ & $1.70(0.37)$ & $1.01(0.19)$ & $1.55(0.37)$ & $31.21^{*}$ & .68 \\
Movement time $(\mathrm{sec})$ & $1.64(0.21)$ & $2.66(0.51)$ & $1.86(0.34)$ & $74.56^{*}$ & .80 \\
\hline
\end{tabular}

$*$ indicates a significant main effect of Condition $(p<.001)$.

\subsection{Movement time}

The one-way repeated measures ANOVA showed a significant main effect of Condition, $F(2$, 38) $=74.56, p<.001, \eta \mathrm{p} 2=.797$. Post-hoc pairwise comparisons revealed participants performed slowest during the task-related condition $(p<.001)$, and fastest during the control condition ( $p s=$ $.005)$.

\subsection{Peak acceleration}

A one-way repeated measures ANOVA showed a significant main effect of Condition for both the reach, $F(2,32)=34.38, p<.001, \eta \mathrm{p} 2=.862$, and transport, $F(2,30)=31.21, p<.001, \eta \mathrm{p} 2=$ .675 , phases of the task. Pairwise comparisons showed peak acceleration to be lower for the taskrelated condition, compared to control and task-unrelated conditions for both reach $(p<.001)$ and transport $(p<.001)$ task phases (Figure 2). 


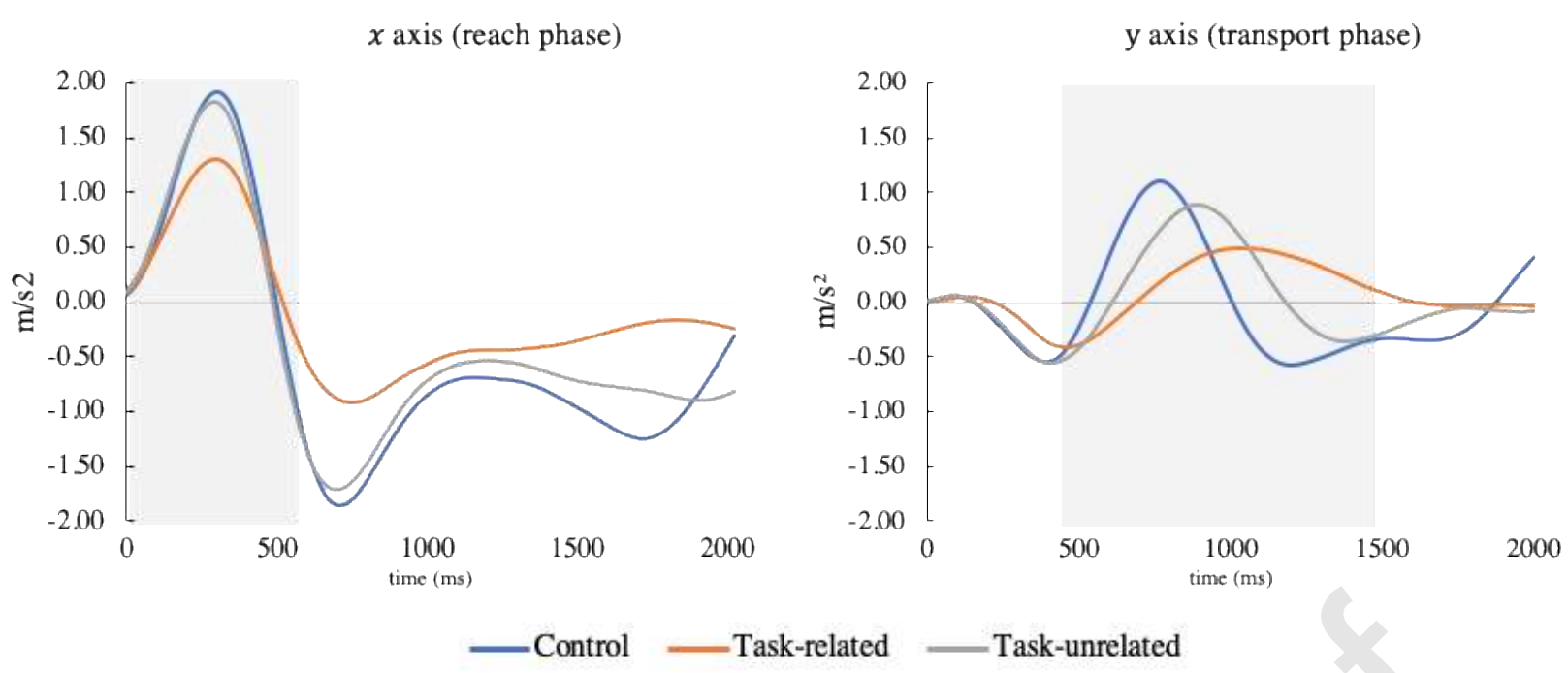

Figure 2. Mean acceleration profiles over time for each condition. Shaded grey areas depict the time window in which peak reach acceleration (left) and peak transport acceleration (right) were identified.

\subsection{Alpha power}

\subsubsection{Left-temporal alpha}

The 3 (Condition) $\times 4$ (Epoch) repeated measures ANOVA conducted on the averaged activity recorded from channels T7, F7 and FC5 yielded a significant main effect of Epoch, $F(1.59$, $30.12)=4.276, p=.031, \eta p 2=.183$, and a significant main effect of Condition, $F(2,38)=4.805, p=$ $.014, \eta p 2=.202$. The Condition $\times$ Epoch interaction was non-significant, $F(6,114)=0.295, p=.938$, $\eta \mathrm{p} 2=.015$. Post-hoc pairwise comparisons to interrogate the main effects revealed that left-temporal alpha power decreased from $M^{+1}$ to $M^{+2}(p=.001)$. They also revealed lower left-temporal alpha power in the task-related self-talk condition, compared to the control $(p=.042)$ and task-unrelated self-talk $(p=.006)$ conditions.

\subsubsection{Regional alpha}

These analyses were conducted to examine topographical effects involving multiple regions of interest covering the major areas of the cerebral cortex, including, but not limited to, the lefttemporal region. Results from a $3($ Condition $) \times 4($ Epoch $) \times 7($ ROI $)$ repeated measures ANOVA yielded a main effect of Condition, $F(2,38)=19.49, p<.001, \eta \mathrm{p} 2=.506$, with significantly lower alpha power observed for the task-related condition $(M=-0.622, S D=0.059)$ compared to the control 
$(M=-0.252, S D=0.046)$ and task-unrelated $(M=-0.244, S D=0.052)$ conditions. There was also a significant main effect of ROI, $F(3.38,64.26)=24.426, p<.001, \eta p 2=.562$, indicating that alpha power was highest over the occipital region, lower over the temporal and frontal regions, and lowest over the central and parietal regions. There were also a significant main effect of Epoch, $F(1.64$, $31.13)=20.28, p<.001, \eta \mathrm{p} 2=.516$, that was superseded by a significant $\mathrm{ROI} \times$ Epoch interaction, $F(7.64,145.17)=7.71, p<.001, \eta p 2=.289$. Pairwise comparisons revealed a significant increase in alpha power over frontal $(p=.002)$ and occipital regions $(p=.001)$ from $P^{-1}$ to $M^{+1}$, which was

followed by a significant decrease in alpha power across all cortical regions from $M^{+1}$ to $M^{+2}$ (rate of change $\left(\left(\mu \mathrm{V}^{2}\right): \mathrm{Fr}=1.975, \mathrm{LT}=0.836, \mathrm{LC}=1.228, \mathrm{RC}=1.759, \mathrm{RT}=1.158, \mathrm{~Pa}=1.005, \mathrm{Oc}=\right.$ 1.393). The interactions for ROI $\times$ Condition, $F(12,228)=0.712, p=.739, \eta p 2=.036$, Condition $\times$ Epoch, $F(6,114)=0.747, p=.613, \eta p 2=.038$, and ROI $\times$ Condition $\times$ Epoch, $F(36,684)=0.819, p$ $=.767, \eta p 2=.041$, were non-significant (see Figure 3). 
$-1.50$

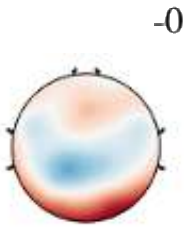

Control

Task-
related

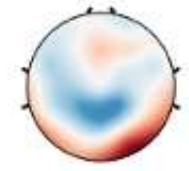

Taskunrelated
$-0.75$
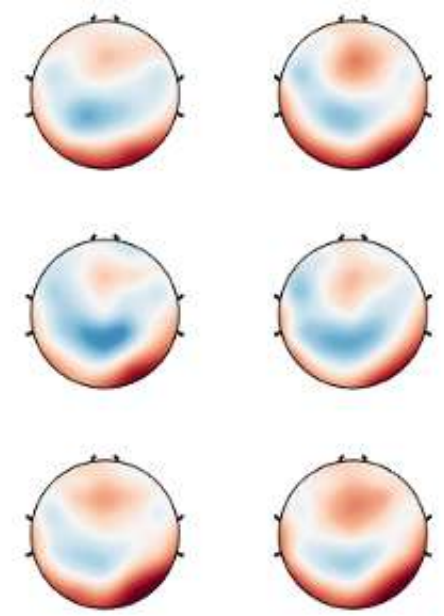

$$
P^{-2}
$$

$$
P^{-1}
$$

0
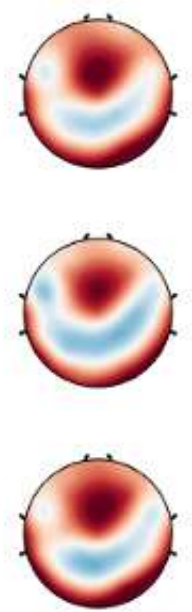
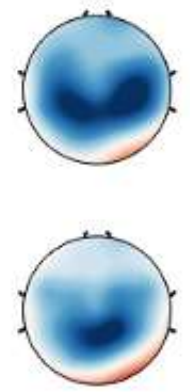

0.75
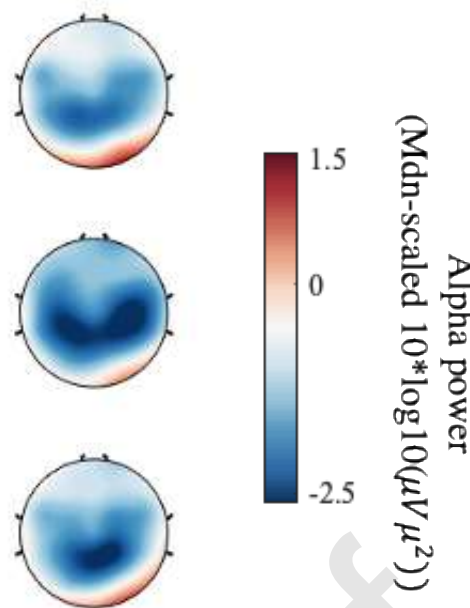
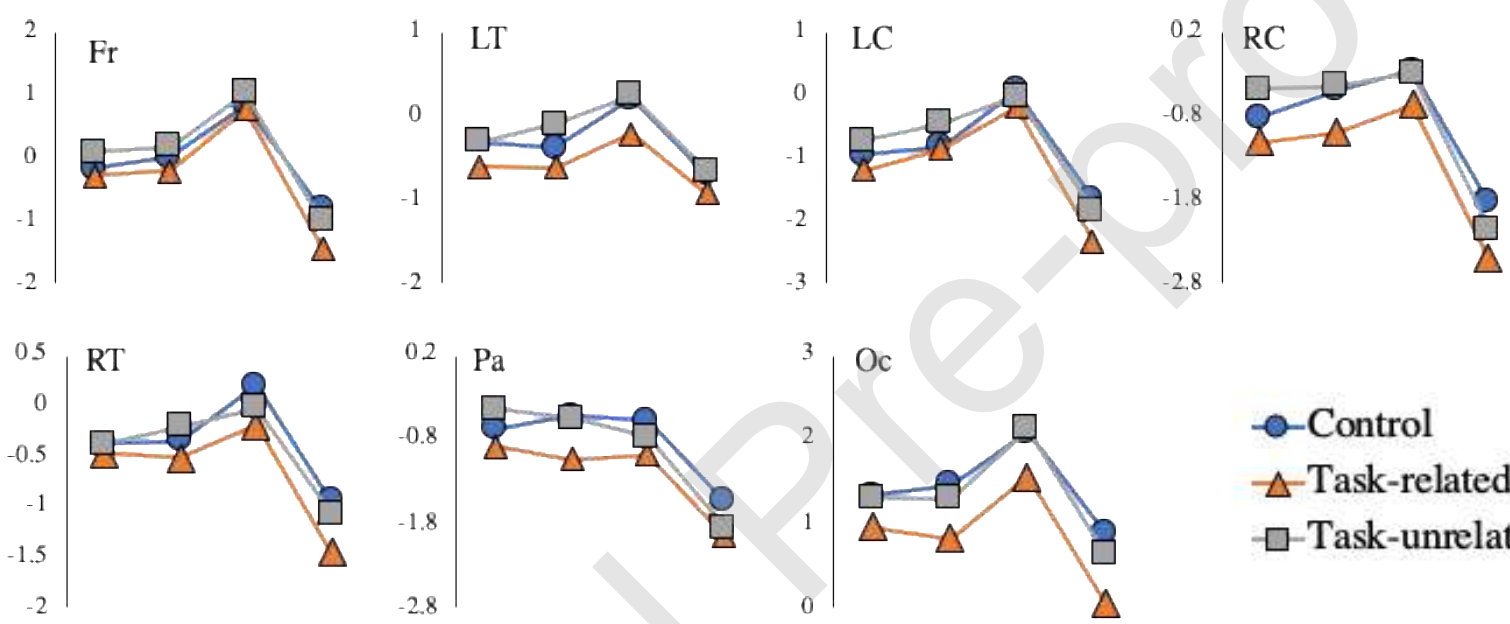

Control

$\triangle$ Task-related

Task-unrelated

Figure 3. Scalp topographies (top) displaying alpha power across Epoch $\left(P^{-2}, P^{-1}, M^{+1}, M^{+2} ; 0=\right.$ "go" signal) for each experimental condition. Line plots (bottom) display the mean alpha power for each region of interest (left temporal $=\mathrm{LT}$, frontal $=\mathrm{Fr}$, left central $=\mathrm{LC}$, right central $=\mathrm{RC}$, Parietal $=\mathrm{Pa}$, right temporal $=\mathrm{RT}$, occipital $=\mathrm{Oc}$ ) as a function of Epoch .

\subsection{Alpha connectivity}

A $2($ Hemisphere $) \times 3($ Condition $) \times 4($ Epoch $)$ repeated measures ANOVA yielded a significant main effect of Hemisphere, $F(1,19)=10.40, p=.004, \eta p 2=.354$, with higher levels of connectivity between T8-Fz $(M=0.25, S D=0.015)$ compared to T7-Fz $(M=0.20, S D=0.012)$. The main effects of Condition, $F(1.45,27.69)=0.128, p=.880, \eta p 2=.007$, and Epoch, $F(1.44,27.39)=$ 
$3.188, p=.071, \eta \mathrm{p} 2=.144$, and the interactions between Hemisphere $\times$ Condition, $F(2,38)=0.306$, $p=.738, \eta \mathrm{p} 2=.016$, Hemisphere $\times$ Epoch, $F(1.97,37.46)=2.170, p=.101, \eta p 2=.103$, Condition $\times$ Epoch, $F(6,114)=0.990, p=.436, \eta p 2=.050$, and Hemisphere $\times$ Condition $\times$ Epoch, $F(6,114)=$ $0.866, p=.522, \eta \mathrm{p} 2=.044$, were all non-significant.
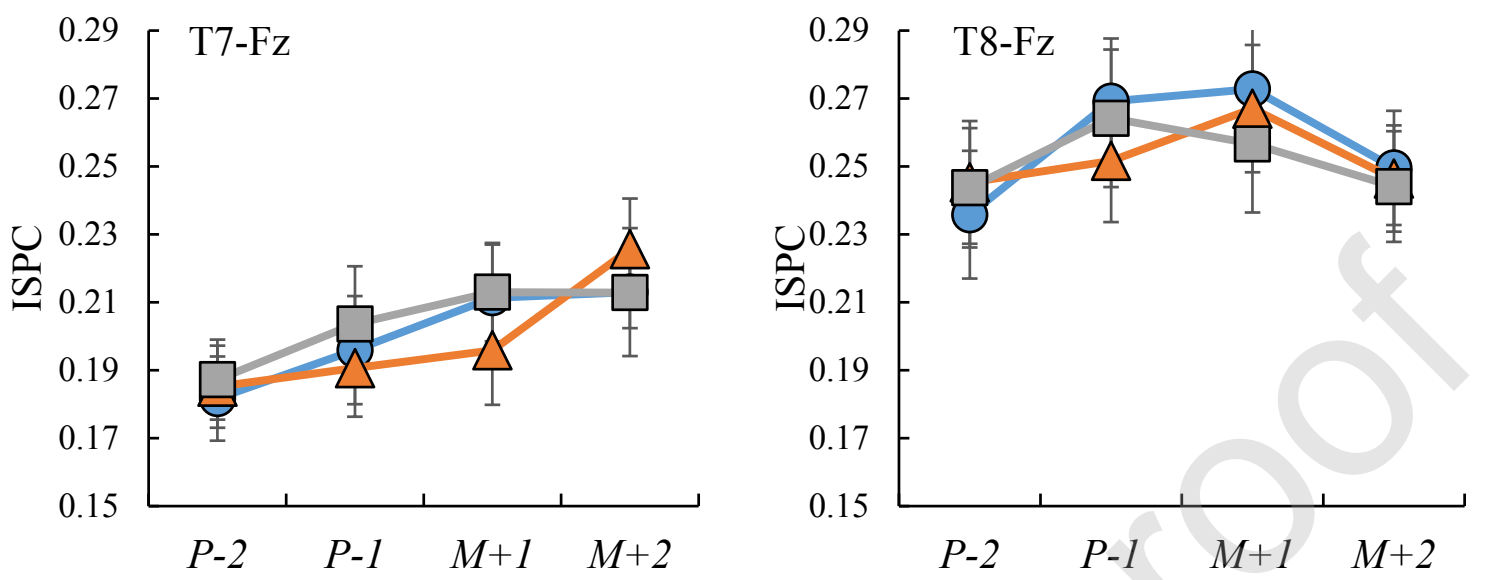

- -Control $\triangle-$ Task-related $-\square-$ Task-unrelated

Figure 4. Line plots displaying the mean ( \pm s.e.m) inter site phase clustering (ISPC) as a function of condition and epoch for both T7-Fz (left) and T8-Fz (right).

\section{Discussion}

The present study attempted to manipulate self-talk during a motor task to evaluate the fidelity of associating EEG left-temporal alpha power and T7-Fz connectivity with verbal processing and conscious motor control. Our results show that both self-talk conditions (task-related and taskunrelated) were rated higher for self-talk frequency and intensity compared to the control condition, confirming that our manipulations increased verbal processing as intended. As predicted, the taskrelated condition was rated as being the most difficult, mentally demanding, and consciously controlled condition. Participants also performed $60 \%$ slower and decreased the speed at which they accelerated the hand when reaching for (30\% slower) and transporting (40\% slower) the jar. This selfreport and behavioural evidence suggest that participants adhered to the task-related verbal instructions and adopted a more conscious mode of motor control. The task-unrelated condition was rated as being more difficult than the control condition, reflected by the slower movement times 
$(\sim 13 \%)$. However, as there was no difference in reported levels of conscious control and mental effort, and no difference in hand acceleration profiles between the task-unrelated and control conditions, it can be argued that task-unrelated self-talk increased verbal processing without inducing increased levels of effort and conscious movement control.

Despite both self-talk conditions increasing verbal processing compared to the control condition, EEG data showed activity in the left-temporal region to only increase (i.e., alpha power decreased) for the task-related condition during both the planning and movement phases of our task. The lack of differences between the control and task-unrelated self-talk condition fails to endorse the thesis that left temporal alpha power can reliably index increases in verbal processing during a motor task. Rather, our data suggest that left temporal activity is more likely to reflect processes associated with conscious movement control beyond language alone.

However, to appropriately interpret the increased left temporal alpha activity observed during the task-related self-talk condition, we must acknowledge that this increase was not specific to the left temporal region but was instead evident across all regions of the cortex. Such a finding mirrors previous research that has shown less-skilled performers to exhibit globally lower EEG alpha power (increased activity) compared to their higher-skilled counterparts (Del Percio et al., 2009; Parr et al., 2019), and is proposed to reflect reductions in neural efficiency. Indeed, alpha rhythms are proposed to reflect thalamo-cortical and cortico-cortico loops that facilitate/inhibit the transmission and retrieval of both sensorimotor and cognitive information into the brain (Deeny et al., 2003; Pfurtscheller \& Lopes da Silva, 1999). We therefore suggest that the global decrease in alpha power during the more consciously performed task-related self-talk condition is not reflective of the engagement of verbal processes alone, but rather a multitude of processes that combine to characterise conscious movement control. Such processes are likely to include increased cognitive effort and the greater demands on attentional resources (including kinaesthetic and visual feedback) to process sensory-motor information as the mechanics of movements are monitored and updated online. It is also important to acknowledge that the task-related self-talk condition significantly altered movement kinematics. As such, it is difficult to untangle the extent to which the present effect is driven by 
changes in neural efficiency, movement efficiency, or an interaction of these factors. However, such a distinction is perhaps not necessary or possible during dynamic motor tasks, given their shared association with the effortful and conscious motor control that characterise the early stages of learning (Fitts \& Posner, 1967).

While previous literature has positioned upper-alpha T7-Fz connectivity as an index of conscious motor processing (Gallicchio, Cooke, \& Ring, 2016; Parr et al., 2019; Zhu, Poolton, Wilson, Hu, et al., 2011), we found no clear association between T7-Fz connectivity and verbal processing or conscious motor control. Indeed, these findings remained consistent even after the application of a surface Laplacian spatial filter to attenuate the effects of volume conduction (Appendix 3). Given that phase-based connectivity reflects the functional coupling between two regions, greater connectivity would be expected between the verbal and motor planning regions if movements were informed by verbal activity, as was achieved here. As this was not the case, the results from this study therefore challenge the previously held interpretation of $\mathrm{T} 7-\mathrm{Fz}$ connectivity in motor tasks.

It could be argued that the type of instructions that we manipulated lacked ecological validity and may not reflect the way that performers use explicit, verbal knowledge to consciously control their movements in other settings. However, these instructions are similar in nature to those used in similar studies that have manipulated internally focused attention (e.g., Ellmers et al., 2016, Chow, Ellmers, Young, Mak \& Wong, 2019) and explicit learning instruction (e.g., Parr et al., 2019), which did report increased T7-Fz connectivity during conscious motor control. The main difference is that in this study we directly manipulated verbal processing and made behavioural (hand acceleration data) and self-report checks to ensure participants were actually engaged in rehearsing task-related explicit knowledge - rather than inferring this from EEG left-temporal activity alone. It could also be argued that our task may not have been novel or difficult enough to induce the non-essential cortico-cortical communication between task-relevant and task-irrelevant cortical regions. However, such a position may further implicate changes in left temporal frontal connectivity as a by-product of more broad changes in cognitive effort rather than specifically reflecting verbal and/or conscious processing. 
Future research should explore the functional communication between verbal and motor planning using a similar methodology but with increased task difficulty.

It should, however, be reiterated that phase-based connectivity merely measures the phase lag consistency between signals recorded from two sites, with relations drawn to communication pathways being inferred rather than directly assessed (Bellomo et al., 2018; Cohen, 2014). As such, it also becomes difficult to interpret our finding that T8-Fz connectivity was significantly higher than T7-Fz connectivity for all conditions across the entirety of our task. Previously, researchers have again inferred the involvement of visuospatial processes in motor performance from T8-Fz connectivity (Bellomo et al., 2018; Cooke, 2013; van Duijn, Buszard, Hoskens, \& Masters, 2017). It could therefore be argued that this observed hemispheric asymmetry reflects a dominant visuospatial component in our reaching and grasping task. However, attempts to directly assess the relationship between T8-Fz alpha connectivity and visuospatial processing during motor performance are required to investigate these claims, similar to how we examined the involvement of T7-Fz connectivity with verbal processing in this study. It could also be argued that these hemispheric differences may be driven by the right-handed nature of our task, given activation of the motor cortex usually shows contralateral bias during upper-limb control (Halsband \& Lange, 2006; Kim et al., 1993). Future work is needed to explore the independent contribution of task laterality to measures of EEG corticocortical connectivity.

In light of our findings, several limitations should be considered. First, it could be argued that our results are limited by administering our control condition in a fixed (always first) order. However, we argue this was necessary to optimise the natural characteristics of initial performance and avoid unintentionally inducing more conscious performance and the accrual of declarative task knowledge. Second, the degree of temporal "jitter" evident in our EEG analyses should be noted. To elaborate, our data were segmented into pre-defined epoch lengths relative to the onset of movement (-1.5 to +1.5 seconds) to enable meaningful comparisons to be made between conditions. However, this method fails to guarantee the segmented data represent the same phase of movement (from 0 to +1.5 seconds) on a trial-to-trial basis, especially given the differences in movement time. Although less 
than ideal, the temporal characteristics of our EEG data were highly consistent between conditions, suggesting a minimal effect of such jitter. Finally, our analyses are potentially limited by solely examining alpha-based connectivity to infer cortico-cortical networking. Indeed, it is still unclear how the interpretation of alpha connectivity differs from the interpretation of non-alpha connectivity (e.g. theta, $4-8 \mathrm{~Hz}$ ). Future research could pay greater attention to this issue and include measures of functional connectivity that either include several frequency bands or do not rely on a specific frequency band (e.g., Granger causality, Phase Slope Index).

In conclusion, these findings challenge the widespread assumption regarding the link between verbal activity, conscious motor control, and left-temporal EEG alpha recorded during motor tasks. Specifically, we show that left-temporal alpha power and upper-alpha T7-Fz connectivity do not share a one-to-one mapping with verbal processing demands, and that increases in conscious motor processing may be better described by a more global increase in cortical activity that is not specific to the left-temporal region. This is likely because the multitude of processes that combine to characterise conscious control (e.g., mental effort, visual processing, muscular effort) reflect a spatially broader phenomenon consistent with decreased neural efficiency. While we are not disputing the role of verbal processing during motor learning or its relationship to conscious movement control, we are challenging the widespread assumption that left-temporal alpha oscillations are a unique index of these processes. We therefore encourage researchers to exercise caution when making such assumptions and to consider EEG activity across the whole topography during the examination of the conscious control of human movement. 


\section{References}

Babiloni, C., Marzano, N., Infarinato, F., Iacoboni, M., Rizza, G., Aschieri, P., ... Del Percio, C. (2010). "Neural efficiency" of experts' brain during judgment of actions: A high-resolution EEG study in elite and amateur karate athletes. Behavioural Brain Research, 207(2), 466475. https://doi.org/10.1016/j.bbr.2009.10.034

Baldauf, D., \& Deubel, H. (2010). Attentional landscapes in reaching and grasping. Vision Research, 50(11), 999-1013. https://doi.org/10.1016/j.visres.2010.02.008

Baumeister, J., Reinecke, K., Liesen, H., \& Weiss, M. (2008). Cortical activity of skilled performance in a complex sports related motor task. European Journal of Applied Physiology, 104(4), 625-631. https://doi.org/10.1007/s00421-008-0811-x

Beauchamp, M. S., Lee, K. E., Argall, B. D., \& Martin, A. (2004). Integration of Auditory and Visual Information about Objects in Superior Temporal Sulcus. Neuron, 41(5), 809-823. https://doi.org/10.1016/S0896-6273(04)00070-4

Bellomo, E., Cooke, A., \& Hardy, J. (2018). Chunking, conscious processing, and EEG during sequence acquisition and performance pressure: A comprehensive test of reinvestment theory. Journal of Sport \& Exercise Psychology, 40(3), 135-145.

Chow, V. W., Ellmers, T. J., Young, W. R., Mak, T. C., \& Wong, T. W. (2019). Revisiting the relationship between internal focus and balance control in young and older adults. Frontiers in neurology, 9, 1131.

Cohen, M. X. (2015). Effects of time lag and frequency matching on phase-based connectivity. Journal of Neuroscience Methods, 250, 137-146. https://doi.org/10.1016/j.jneumeth.2014.09.005

Cohen, M. X. (2014). Analyzing Neural Time Series Data: Theory and Practice. MIT Press.

Cooke, A. (2013). Readying the head and steadying the heart: a review of cortical and cardiac studies of preparation for action in sport. International Review of Sport and Exercise Psychology, 6(1), 122-138. https://doi.org/10.1080/1750984X.2012.724438 
Corcoran, A. W., Alday, P. M., Schlesewsky, M., \& Bornkessel-Schlesewsky, I. (2018). Toward a reliable, automated method of individual alpha frequency (IAF) quantification. Psychophysiology, 55(7), e13064. https://doi.org/10.1111/psyp.13064

Deeny, S. P., Hillman, C. H., Janelle, C. M., \& Hatfield, B. D. (2003). Cortico-cortical communication and superior performance in skilled marksmen: An EEG coherence analysis. Journal of Sport \& Exercise Psychology, 25(2), 188-204.

Del Percio, C., Babiloni, C., Bertollo, M., Marzano, N., Iacoboni, M., Infarinato, F., ... Eusebi, F. (2009). Visuo-attentional and sensorimotor alpha rhythms are related to visuo-motor performance in athletes. Human Brain Mapping, 30(11), 3527-3540. https://doi.org/10.1002/hbm.20776

Delorme, A., \& Makeig, S. (2004). EEGLAB: an open source toolbox for analysis of single-trial EEG dynamics including independent component analysis. Journal of Neuroscience Methods, 134(1), 9-21. https://doi.org/10.1016/j.jneumeth.2003.10.009

Desmurget, M., \& Grafton, S. (2000). Forward modelling allows feedback control for fast reaching movements. Trends in Cognitive Sciences, 4(11), 423-431.

Ellmers, T. J., Machado, G., Wong, T. W.-L., Zhu, F., Williams, A. M., \& Young, W. R. (2016). A validation of neural co-activation as a measure of attentional focus in a postural task. Gait \& Posture, 50, 229-231. https://doi.org/10.1016/j.gaitpost.2016.09.001

Faul, F., Erdfelder, E., Buchner, A., \& Lang, A. G. (2009). Statistical power analyses using G* Power 3.1: Tests for correlation and regression analyses. Behavior research methods, 41(4), 11491160.

Fitts, P. M., \& Posner, M. I. (1967). Human performance. Belmont, Calif.: Brooks/Cole Pub. Co. Gallicchio, G., Cooke, A., \& Ring, C. (2016). Lower left-temporal-frontal connectivity characterizes expert and accurate performance: High-alpha T7-Fz connectivity as a marker of conscious processing during movement. Sport, Exercise, and Performance Psychology, 5(1), 14.

Gallicchio, G., Cooke, A., \& Ring, C. (2017). Practice makes efficient: Cortical alpha oscillations are associated with improved golf putting performance. Sport, Exercise, and Performance Psychology, 6(1), 89-102. https://doi.org/10.1037/spy0000077 
Gallicchio, G., Finkenzeller, T., Sattlecker, G., Lindinger, S., \& Hoedlmoser, K. (2016). Shooting under cardiovascular load: Electroencephalographic activity in preparation for biathlon shooting. International Journal of Psychophysiology, 109, 92-99. https://doi.org/10.1016/j.ijpsycho.2016.09.004

Halsband, U., \& Lange, R. K. (2006). Motor learning in man: a review of functional and clinical studies. Journal of Physiology-Paris, 99(4-6), 414-424.

Harmon- Jones, E. (2006). Unilateral right- hand contractions cause contralateral alpha power suppression and approach motivational affective experience. Psychophysiology, 43(6), 598603.

Hatfield, B. D. (2018). Brain dynamics and motor behavior: A case for efficiency and refinement for superior performance. Kinesiology Review, 7(1), 42-50. https://doi.org/10.1123/kr.2017-0056

Hatfield, B. D., \& Hillman, C. H. (2001). The psychophysiology of sport: A mechanistic understanding of the psychology of superior performance. 2, 362-386.

Haufler, A. J., Spalding, T. W., Santa Maria, D. L., \& Hatfield, B. D. (2000). Neuro-cognitive activity during a self-paced visuospatial task: comparative EEG profiles in marksmen and novice shooters. Biological Psychology, 53(2), 131-160. https://doi.org/10.1016/S03010511(00)00047-8

Janelle, C. M., Hillman, C. H., Apparies, R. J., Murray, N. P., Meili, L., Fallon, E. A., \& Hatfield, B. D. (2000). Expertise differences in cortical activation and gaze behavior during rifle shooting. Journal of Sport and Exercise Psychology, 22(2), 167-182. https://doi.org/10.1123/jsep.22.2.167

Jensen, O., \& Mazaheri, A. (2010). Shaping functional architecture by oscillatory alpha activity: Gating by inhibition. Frontiers in Human Neuroscience, 4. https://doi.org/10.3389/fnhum.2010.00186

Kerick, S. E., Douglass, L. W., \& Hatfield, B. D. (2004). Cerebral cortical adaptations associated with visuomotor practice. Medicine and Science in Sports and Exercise, 36(1), 118-129. https://doi.org/10.1249/01.MSS.0000106176.31784.D4 
Kim, S. G., Ashe, J., Hendrich, K., Ellermann, J. M., Merkle, H., Ugurbil, K., \& Georgopoulos, A. P. (1993). Functional magnetic resonance imaging of motor cortex: hemispheric asymmetry and handedness. Science, 261(5121), 615-617.

Klimesch, W. (2012). Alpha-band oscillations, attention, and controlled access to stored information. Trends in Cognitive Sciences, 16(12), 606-617. https://doi.org/10.1016/j.tics.2012.10.007

Lachaux, J.-P., Rodriguez, E., Martinerie, J., \& Varela, F. J. (1999). Measuring phase synchrony in brain signals. Human Brain Mapping, 8(4), 194-208. https://doi.org/10.1002/(SICI)10970193(1999)8:4<194::AID-HBM4>3.0.CO;2-C

Masters, R. S. W. (1992). Knowledge, nerves and know-how: The role of explicit versus implicit knowledge in the breakdown of a complex motor skill under pressure. British Journal of Psychology, 83(3), 343-358. https://doi.org/10.1111/j.2044-8295.1992.tb02446.x

Parr, J. V. V., Vine, S. J., Wilson, M. R., Harrison, N. R., \& Wood, G. (2019). Visual attention, EEG alpha power and T7-Fz connectivity are implicated in prosthetic hand control and can be optimized through gaze training. Journal of NeuroEngineering and Rehabilitation, 16(1), 52. https://doi.org/10.1186/s12984-019-0524-x

Parr, J. V. V. (2018). Evaluating and alleviating the cognitive burden associated with myoelectric prosthetic hand control (Doctoral dissertation, Liverpool Hope University).

Pfurtscheller, G., \& Lopes da Silva, F. H. (1999). Event-related EEG/MEG synchronization and desynchronization: basic principles. Clinical Neurophysiology, 110(11), 1842-1857. https://doi.org/10.1016/S1388-2457(99)00141-8

Poldrack, R. A. (2006). Can cognitive processes be inferred from neuroimaging data? Trends in Cognitive Sciences, 10(2), 59-63. https://doi.org/10.1016/j.tics.2005.12.004

Tiihonen, J., Hari, R., Kajola, M., Karhu, J., Ahlfors, S., \& Tissari, S. (1991).

Magnetoencephalographic $10-\mathrm{Hz}$ rhythm from the human auditory cortex. Neuroscience Letters, 129(2), 303-305. https://doi.org/10.1016/0304-3940(91)90486-D

van Duijn, T., Buszard, T., Hoskens, M. C. J., \& Masters, R. S. W. (2017). Chapter 13 - Discerning measures of conscious brain processes associated with superior early motor performance: 
Capacity, coactivation, and character. In M. R. Wilson, V. Walsh, \& B. Parkin (Eds.), Progress in Brain Research (pp. 245-261). https://doi.org/10.1016/bs.pbr.2017.06.013

Vingerhoets, G. (2014). Contribution of the posterior parietal cortex in reaching, grasping, and using objects and tools. Frontiers in Psychology, 5. https://doi.org/10.3389/fpsyg.2014.00151

Zhu, F. F., Poolton, J. M., Wilson, M. R., Hu, Y., Maxwell, J. P., \& Masters, R. S. W. (2011). Implicit motor learning promotes neural efficiency during laparoscopy. Surgical Endoscopy, 25(9), 2950. https://doi.org/10.1007/s00464-011-1647-8

Zhu, F. F., Poolton, J. M., Wilson, M. R., Maxwell, J. P., \& Masters, R. S. W. (2011). Neural coactivation as a yardstick of implicit motor learning and the propensity for conscious control of movement. Biological Psychology, 87(1), 66-73. https://doi.org/10.1016/j.biopsycho.2011.02.004

\section{ORCID}

Johnny V. V. Parr - https://orcid.org/0000-0002-3096-2601

\section{Conflict of interest}

No conflicting interests are declared.

\section{Footnotes}

1. Sites $\mathrm{T} 7$ and $\mathrm{T} 8$ are also referred to as $\mathrm{T} 3$ and $\mathrm{T} 4$, respectively, in older EEG systems. 


\section{Appendix 1: Manipulations to ensure adherence to task-related self-talk instructions}

Photos depicting the placement of electrodes both on the workspace and arm to encourage full adherence to the task-related self-talk instructions. Manipulations were designed to encourage a more conscious mode of movement control.

\section{"Keep hand $5 \mathrm{~cm}$ above table"}

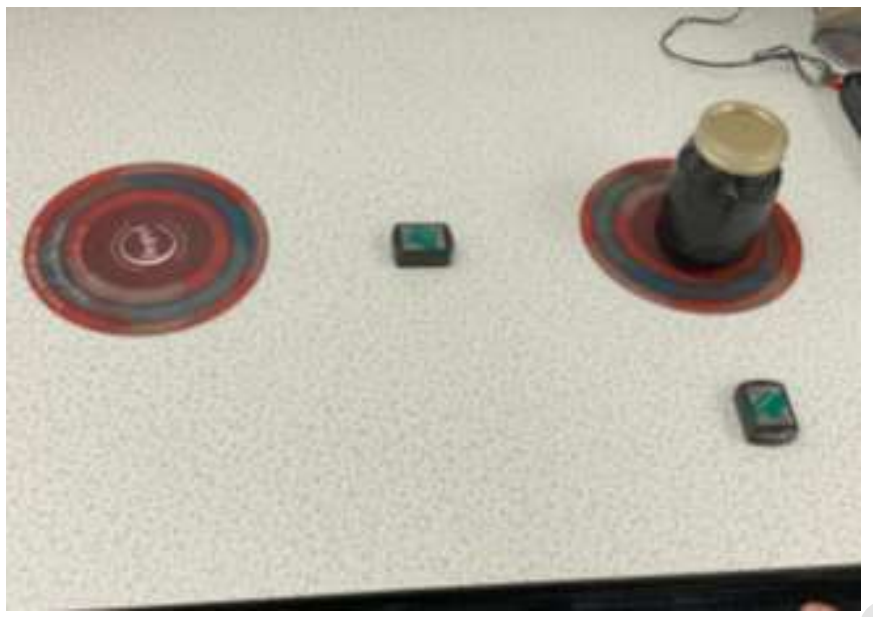

Two additional electrodes were placed halfway between the hand start location and the jar, and halfway between the jar and target locations. Participants were informed that the distance between the electrode on hand and the electrodes on the workspace would be recorded during performance. As such, participants were instructed to ensure this distance was as close to $5 \mathrm{~cm}$ as possible.

\section{"Keep elbow below the wrist"}

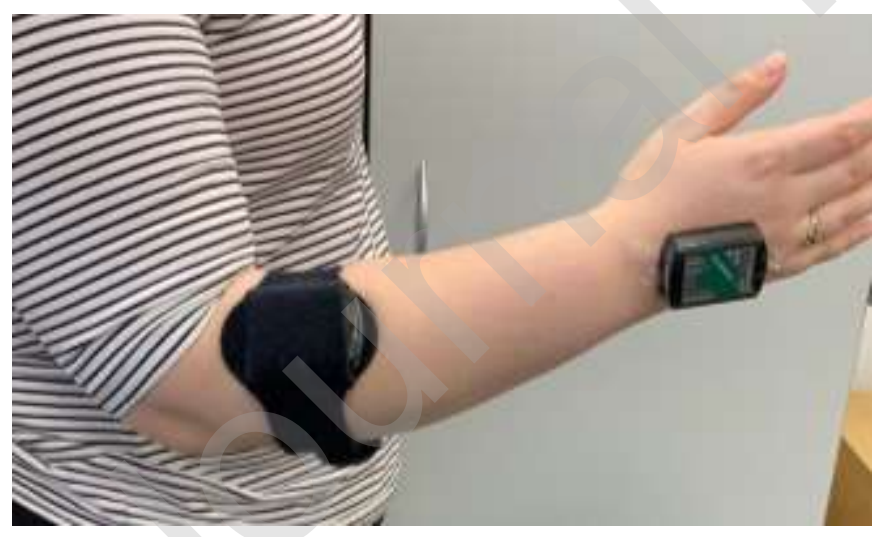

An additional electrode was placed towards the elbow using a Velcro strap. Participants were informed that the electrode on the elbow must remain below the electrode on the hand throughout task performance. Participants were told this would be objectively assessed.

“Keep wrist flexed at 90 degrees" 


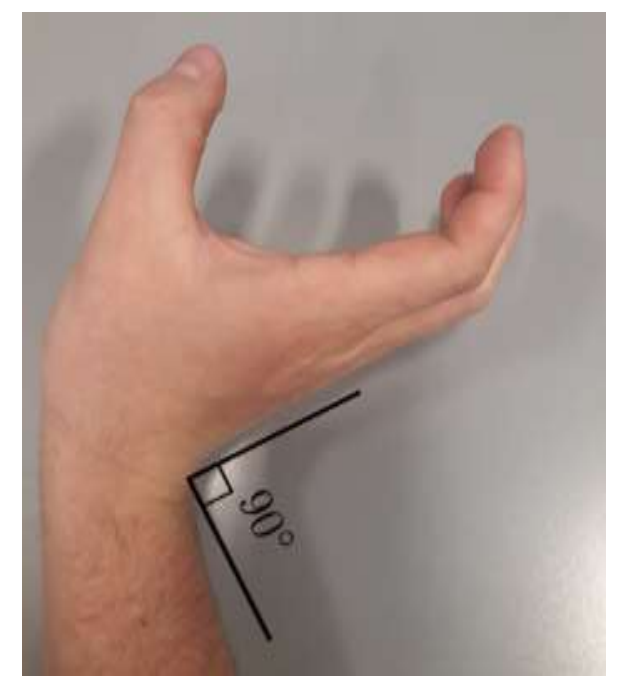

Participants were required to perform the task with their hand hyperextended to create a 90 degree angle with the forearm (pictured). Participants were told that their adherence could be objectively monitored using the electrode placed on the hand (not pictured).

\section{"Keep thumb below the index"}

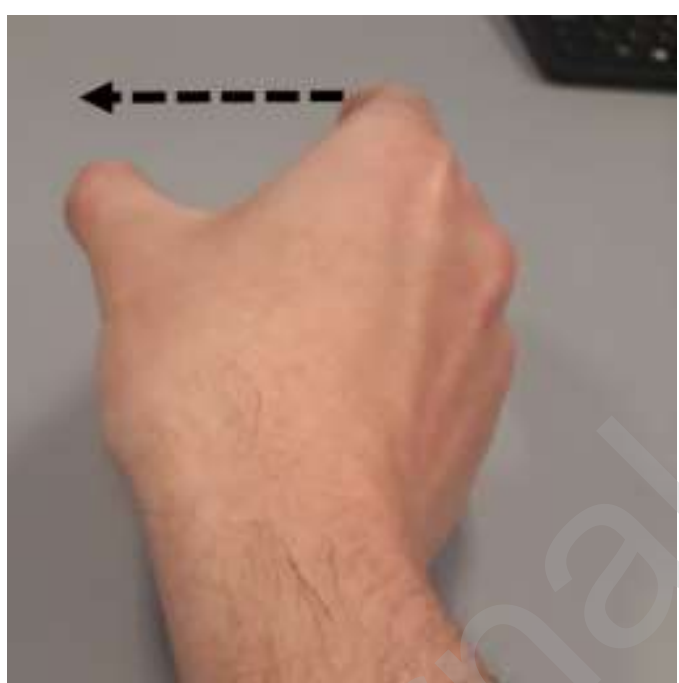

Participants were required to rotate their hand in a manner that ensure the thumb was always positioned lower than the index finger (as opposed to being in-line. Again, participants were told that adherence would be objectively assessed by calculating the angle of the hand electrode during performance (not pictured). 


\section{Appendix 2: Individual alpha frequency identification}

As the actual alpha frequency band can show inter-subject variability, we attempted to specify each participant's individual alpha frequency band (IAF). The below figure displays the mean spectra recorded over occipital-parietal electrodes during a 1-minute eyes closed resting baseline. For the line plots that are in red, IAF was taken as the peak frequency occurring between 7 and $15 \mathrm{~Hz}$, as a clear single peak could be observed upon inspection within this band. For the line plots that are in black, a clear single peak was not visible, resulting in the IAF being taken as the power spectra weighted mean (centre of gravity) within the $7-15 \mathrm{~Hz}$ band. For one participant, baseline data was corrupt resulting in IAF being set at the typical $10 \mathrm{~Hz}$.
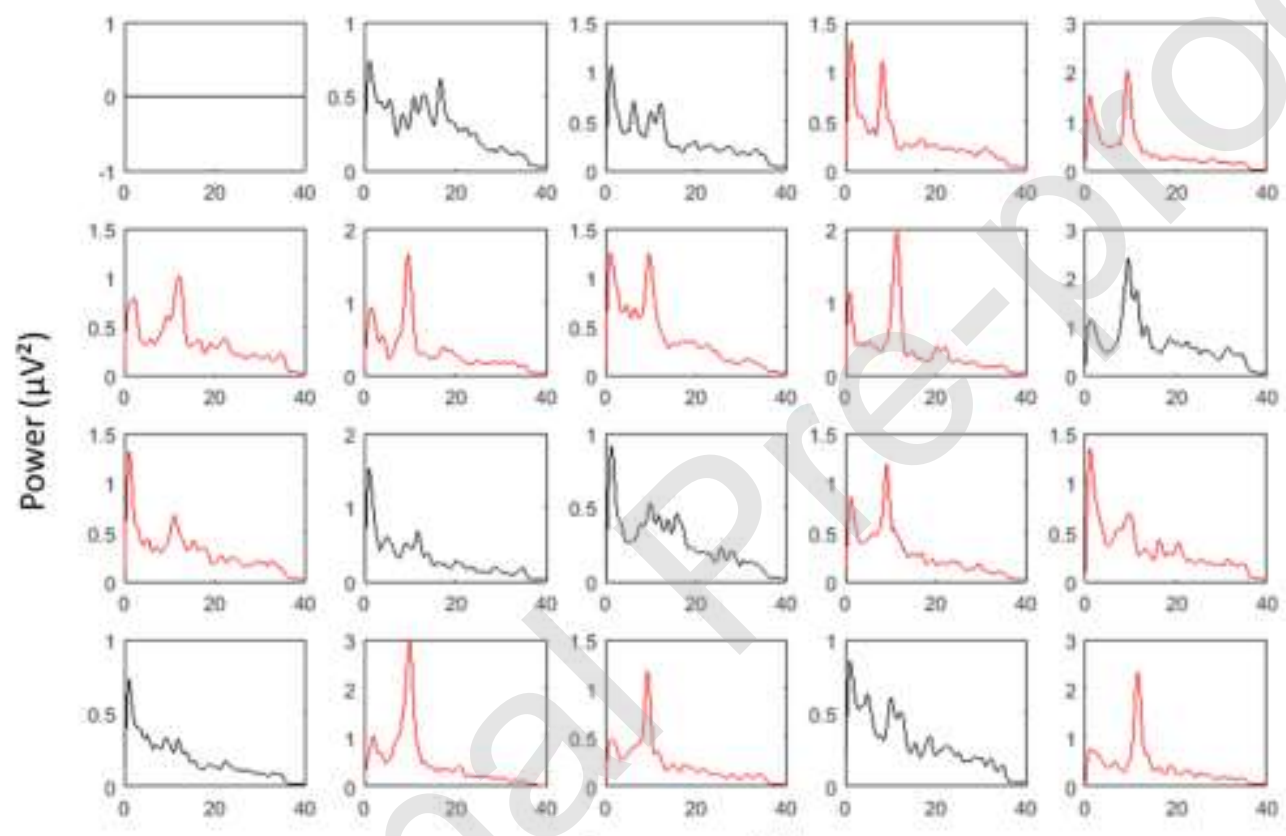

Frequency $(\mathrm{Hz})$

Fig 1. Line plots displaying mean spectral power recorded over occipital-parietal electrodes during a 1-minute eyes closed resting baseline. Each line plot represents a single participant. Red lines indicate the identification of a single peak alpha frequency, whilst black lines indicate the calculation of the centre of gravity. 


\section{Appendix 3: Spatially enhanced analyses of alpha power and connectivity using surface} Laplacian and channel levels analyses.

To enhance the local features of our data, we applied a scalp-level surface Laplacian transformation (Cohen, 2014; function available at http://mikexcohen.com/lectures.html), which acts as a spatial band-pass filter to attenuate the effects of volume conduction. In line with our aim to highlight local features, we conducted analyses for alpha power on a channel level over sites T7, T8, Fz, CP1, CP2, FC1, FC2, O1, O2. These channels were chosen based upon previous literature and topographical inspection of our data (Figure 1).

\section{Results}

\section{Alpha power}

Results from a 3 (Condition) $\times 4$ (Epoch) $\times 9$ (Channel) repeated measures ANOVA showed a significant main effect of Condition, $F(2,38)=8.233, p=.001, \eta \mathrm{p} 2=.302$, a main effect of Epoch, $F(1.598,30.366)=10.927, p=.001, \eta p 2=.365$, and a main effect of Channel, $F(3.79,72.01)=$ $15.435, p<.001, \eta p 2=.448$. These effects were superseded by significant Condition $\times$ Epoch, $F(6$, $114)=2.741, p=.016, \eta \mathrm{p} 2=.126$, and Epoch $\times$ Channel, $F(24,456)=6.813, p<.001, \eta p 2=.264$, interactions. Due to the aim of this analysis, we only further examined effects involving the factor Channel. For the Epoch $\times$ Channel interaction, Bonferroni pairwise comparisons revealed that alpha power significantly increased over channels T7 $(p=.010)$, T8 $(p=.023)$, CP2 $(p=.050)$, and FC1 ( $p$ $=, 001)$ from $P^{-1}$ to $M^{+1}$. Alpha power then significantly decreased from $M^{+1}$ to $M^{+2}$ over channels Fz $(p=.005), \mathrm{T} 7(p=.048), \mathrm{T} 8(p=.001), \mathrm{CP} 1(p=.002), \mathrm{CP} 2(p<.001), \mathrm{FC} 1(p<.001)$ and FC2 $(p<$ $.001)$. Interestingly, alpha activity over occipital electrodes did not change over time ( $p s=.404)$. 


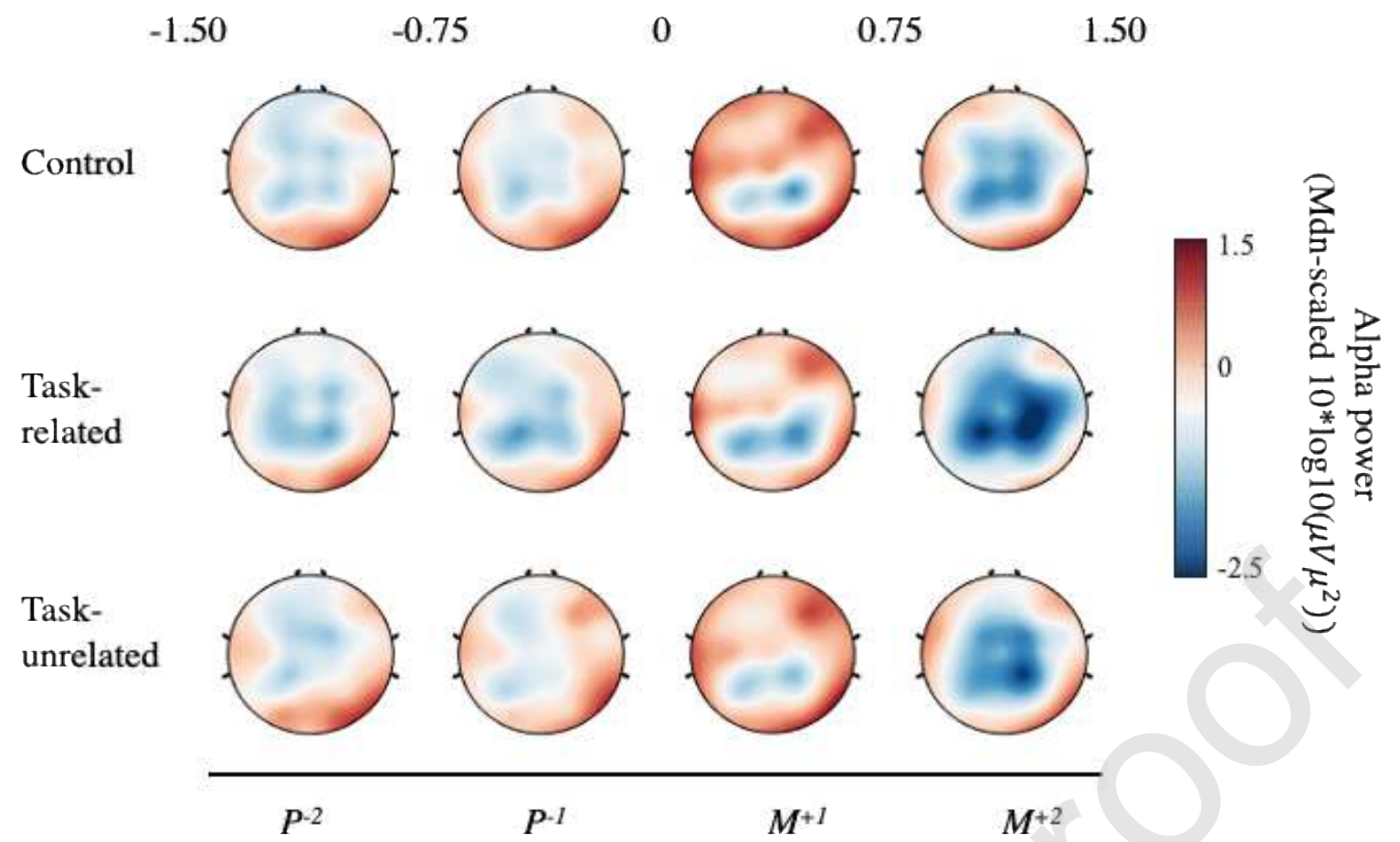

Figure 1. Scalp topographies depicting surface Laplacian spatially enhanced regional alpha power across time, relative to movement onset, for each experimental condition.

\section{Alpha connectivity}

A 3 (Condition) $\times 2$ (Hemisphere $) \times 4($ Epoch $)$ repeated measures ANOVA showed no main effect of Hemisphere, $F(1,19)=0.650, p=.430, \eta p 2=.003$, Condition, $F(2,38)=0.656, p=.525$, $\eta \mathrm{p} 2=.033$, or Epoch, $F(3,57)=0.133, p=.940, \eta \mathrm{p} 2=.007$. There was however a significant Hemisphere $\times$ Epoch interaction, $F(2.184,41.490)=4.100, p=.021, \eta p 2=.177$. Pairwise comparisons revealed greater T8-Fz connectivity $(M=0.188, S D=.012)$ compared to T7-Fz connectivity $(M=0.165, S D=.006)$ at epoch $P^{-2}$, though this failed to reach significance $(p=.099)$.
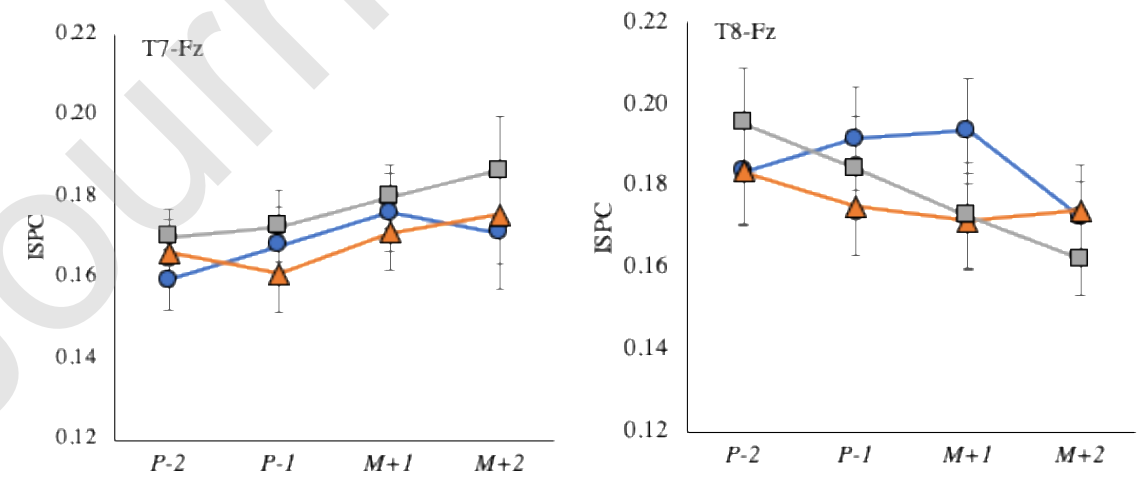

Fig 2. Line plots displaying the mean ( \pm s.e.m) intersite phase clustering (ISPC) as a function of condition and epoch for both T7-Fz (left) and T8-Fz (right). 